Relations industrielles

Industrial Relations

\title{
Jacques W. Serruys : Phychologie du commandement. Bruxelles, Vander Éditeur, 1984, 230 pp., ISBN 2-8008-0000-3
}

\section{Hélène Bois-Brochu}

Volume 40, numéro 2, 1985

URI : https://id.erudit.org/iderudit/050151ar

DOI : https://doi.org/10.7202/050151ar

Aller au sommaire du numéro

Éditeur(s)

Département des relations industrielles de l'Université Laval

ISSN

0034-379X (imprimé)

1703-8138 (numérique)

Découvrir la revue

Citer ce compte rendu

Bois-Brochu, H. (1985). Compte rendu de [Jacques W. Serruys : Phychologie du commandement. Bruxelles, Vander Éditeur, 1984, 230 pp., ISBN 2-8008-0000-3] Relations industrielles / Industrial Relations, 40(2), 414-415.

https://doi.org/10.7202/050151ar

Tous droits réservés @ C Département des relations industrielles de l'Université Laval, 1985
Ce document est protégé par la loi sur le droit d'auteur. L'utilisation des services d'Érudit (y compris la reproduction) est assujettie à sa politique d'utilisation que vous pouvez consulter en ligne.

https://apropos.erudit.org/fr/usagers/politique-dutilisation/ 
niveau de la description et de la compilation; l'analyse sociologique des professions est suffisammient développée pour fournir à l'historien des cadres pour analyser et donner une signification aux faits qu'il reconstitue. Faute de cadre analytique, Hamowy a négligé toutes sortes de questions dont l'examen pourrait donner un éclairage plus vif du processus de monopolisation des pratiques sanitaires par les médecins. Quelles étaient les origines sociales des membres des nombreux groupes qui se disputaient le contrôle du marché des soins? Comment un marché s'estil constitué et développé? Quels étaient les liens de chaque groupe avec les pouvoirs politiques? Quels appuis les médecins ont-ils réussi à obtenir pour supporter leurs demandes restrictives? En somme, pourquoi les médecins, plutôt que les homéopathes ou les ostéopathes ou tout autre groupe, ont-ils réussi dans leur quête de privilèges? Peut-être ces questions débordaient-elles les limites que Hamowy s'était données; sa contribution à l'étude de l'émergence des monopoles occupationnels n'en demeure pas moins importante et devrait stimuler l'intérêt des chercheurs-historiens, économistes, sociologues - pour l'analyse socio-historique des occupations.

\section{Gilles DUSSAULT}

Université Laval

Psychologie du commandement, par Jacques W. Serruys, Vander éditeur, Bruxelles, 1984, 230 pp., ISBN 2-8008-0000-3

Dans cet ouvrage destiné aux gestionnaires de tous niveaux et de tous genres d'entreprises, Jacques Serruys expose les grands principes gouvernant l'art d'exercer I'autorité. En se basant sur son expérience personnelle et sur les différentes théories de la psychologie sociale, il explique comment un chef devrait exercer son commandement, selon les circonstances, de façon à augmenter l'efficacité de son groupe de travail et à y assurer la régulation de l'autorité. L'auteur utilise à cette fin un langage clair et d'une compréhen- sion aisée. Il expose les théories en question de façon simple et pratique de sorte que les lecteurs peuvent les appliquer facilement.

Bien que l'ouvrage n'ajoute rien de nouveau aux sciences du comportement, il a l'avantage de faire une bonne synthèse des notions fondamentales de gestion du personnel, de leadership et de dynamique des groupes. Il couvre ainsi les principaux aspects de la fonction d'autorité. Malgré cette approche synthétique, nous retrouvons dans ce livre les nuances et les avertissements de prudence nécessaires à l'exercice judicieux de l'autorité. De plus ce volume peut fort bien constituer une lecture de base dans ce domaine pour tous les cadres qui n'ont encore reçu que très peu de formation en gestion, quel que soit leur niveau d'instruction. Je déplore cependant que la bibliographie contienne peu d'ouvrages récents et ne réfère pas à des lectures qui permettraient d'approfondir certains points qui semblent plus intéressants. Cette lacune n'enlève cependant rien à la valeur des enseignements exposés.

La première partie traite des dimensions nouvelles du commandement. L'auteur s'attaque d'abord au mythe du don inné de leadership et soutient que l'art de diriger les êtres humains peut s'apprendre en se basant sur les données des sciences du comportement. Puis il clarifie les concepts de commandement et le leadership, en soulignant au passage la nécessité pour un chef à la fois d'être accepté par les membres de son équipe et d'être attentif aux besoins et aspirations de cette dernière. De plus, il définit les notions de responsabilité, de pouvoir et d'autorité, et il attire l'attention sur les sources de celle-ci. Ensuite il s'attarde aux styles de commandement, tels que définis par Lewin, Lippitt et White, ct met en évidence les effets de chacun sur le groupe ainsi que la portée de leur expérience, tout en mentionnant l'apport de Fiedler et des autres chercheurs dans ce domaine. Enfin il parle du commandement de type associatif et de la typologie du leadership centré sur le groupe et il fait ressortir les avantages d'un tel style pour améliorer la productivité du groupe de travail. 
Dans la deuxième partie, l'auteur parle du chef et de son équipe. Il expose d'abord les notions fondamentales de la psychologie des groupes restreints. Ceux-ci étant à la base de toute organisation sociale, selon lui, un gestionnaire ne peut pas diriger efficacement sans en connaître le fonctionnement et les effets sur le comportement des membres et sur l'organisation. Ensuite il explique les mécanismes et le rôle de la communication dans les relations interpersonnelles et intergroupes à l'intérieur de l'organisation et il mentionne les principaux problèmes qui y sont reliés. Puis il aborde la question des réunions de travail et du travail en équipe comme mode de fonctionnement visant à augmenter l'efficacité et la créativité du groupe de travail. Il définit aussi les divers types de réunions en soulignant leurs avantages et leurs inconvénients respectifs et propose comme modèle la réunion de collaboration (dont font partie le «task forces》 et les cercles de qualité); de même il trace les grandes lignes du rôle de l'animateur dans un tel groupe, tout en attirant l'attention sur les difficultés qui peuvent surgir dans son fonctionnement et sur les façons de les surmonter. Il fait ensuite remarquer que le groupe n'est pas toujours le meilleur instrument de décision et que le chef doit se réserver cette prérogative dans des situations critiques ou de tensions extrêmes; il lui faut donc reconnaître ces moments et être attentif aux phénomènes de freinage qui peuvent accompagner ces décisions unilatérales. C'est pourquoi il est essentiel de contrôler efficacement l'exécution des ordres donnés. Enfin il parle de la nécessité d'une certaine discipline dans le groupe pour atteindre les objectifs de rendement. Il affirme donc que le chef doit se montrer vigilant et équitable à cet égard et savoir appliquer les sanctions qui s'imposent le cas échéant; car son rôle de leader lui impose de faire appliquer les normes et d'en sanctionner les infractions sous peine de perdre son autorité et le respect des membres du groupe.
La troisième partie du livre s'intéresse au comportement du chef face à chacun de ses collaborateurs. L'auteur y explique que le gestionnaire doit apprendre à les connaître tous, à les accepter tels qu'ils sont et à les encourager pour les motiver à bien travailler. Il met aussi en évidence le rôle de formation que doit jouer un dirigeant pour s'assurer de la bonne exécution des tâches, pour bien intégrer ses subordonnés et les nouveaux venus à l'équipe de travail et à l'organisation et pour ainsi augmenter leur satisfaction provenant d'un bon climat affectif de travail, de même que pour améliorer le rendement de chacun. De plus il souligne la nécessité de savoir déléguer plusieurs tâches aux subordonnés et de ne conserver que les fonctions de gestion. Il aborde ensuite la question des entretiens avec les membres de l'équipe, ces entrevues, dont le type peut varier selon les objectifs poursuivis (sélection, appréciation, etc.), nécessitent de la part du gestionnaire une adaptation de son comportement en fonction de ceux-ci.

Enfin l'auteur trace un portrait du nouveau chef qui n'a plus à se créer un personnage pour réussir, mais que la compétence, l'intérêt qu'il manifeste pour son équipe et l'enthousiasme communicatif rendront plus apte à remplir sa fonction. Celui-ci doit savoir être attentif aux besoins de son groupe et avoir confiance aux personnes qu'il dirige pour leur donner la latitude nécessaire à leur motivation et à leur affirmation personnelle, ce qui devrait se refléter tôt ou tard par une amélioration de leur rendement.

En arrière-propos, l'auteur attire l'attention sur le fait que, généralement, la fonction de commandement n'est pas l'unique tâche du gestionnaire, mais qu'il doit aussi mettre ses compétences professionnelles au service de son propre supérieur. Il s'interroge enfin sur la possibilité, dans ces conditions, de se consacrer pleinement à son rôle de commandement et sur l'éventualité d'une telle possibilité.

Hélène BOIS-BROCHU

Université Laval 